\title{
Nanotecnologia em mídias: utopias e distopias
}

\author{
Nanotechnology in the media: utopias and dystopias
}

\author{
Clecí Körbes ${ }^{1}$ \\ Noela Invernizzi²
}

Artigo recebido em para publicação em set/2013 e aceito para publicação em mar/2014

\section{RESUMO}

Esse artigo examina a abordagem de nanotecnologia em mídias que respondem a interesses e valores de grupos sociais diferentes. Utiliza-se a metodologia de análise de conteúdo com base nos fundamentos dos Estudos Sociais da Ciência e da Tecnologia. Analisam-se as seções de ciência do telejornal Bom Dia Brasil e do jornal Folha de S. Paulo no período de 2008 a 2010, e uma amostra não aleatória de $20 \%$ das entrevistas do programa Nanotecnologia do Avesso, no período de 2009 a 2010. Observou-se que as definições de nanotecnologia variam entre os meios, dependendo de quem as produz e das circunstâncias que levam à sua promulgação e estabilização. No Bom Dia Brasil e na Folha de $S$. Paulo sobressaem expectativas de que as propriedades e funcionalidades da matéria em nanoescala redundem em benefícios, reproduzindo visões lineares de ciência e tecnologia. No Nanotecnologia do Avesso são proeminentes visões distópicas e a demanda pela aplicação do princípio de precaução e regulação obrigatória.

Palavras-chave: Divulgação científica. Tecnologia. Nanotecnologia. Utopias. Distopias.

\section{ABSTRACT}

This article examines the approach to nanotechnology in medias that respond to the interests and values of different social groups. The methodological approach consists of a content analysis, based on the Social Studies of Science and Technology. The article analyzes the science sections of the television program Bom Dia Brasil and the newspaper Folha de S. Paulo during the period 2008-2010, and a non-aleatory $20 \%$ sample of the interviews showed on the program Nanotecnologia do Avesso exhibited during 2009-2010. The comparative analysis showed that definitions of nanotechnology vary among the media, depending on who produced them and the circumstances that led to their enunciation and stabilization. Bom Dia Brasil and Folha de S. Paulo emphasize the promises and benefits of the properties and functionalities of the matter in the nanoscale, reproducing linear visions of science and technology. In Nanotecnologia do Avesso dystopian visions and the demand for the application of the precautionary principle and mandatory regulation are preeminent.

Keywords: Science popularization. Technology. Nanotechnology. Utopias. Dystopias.

\footnotetext{
1 Graduação em Pedagogia e em Administração pela Universidade Federal do Paraná (UFPR), mestrado em Educação pela mesma Universidade e doutorado em Tecnologia pela Universidade Tecnológica Federal do Paraná (UTFPR). Pós-doutoranda em Educação na UFPR, com bolsa da Coordenação de Aperfeiçoamento de Pessoal de Nível Superior (CAPES). E-mail: cleci.korbes@gmail.com

2 Graduação em Antropologia pela Universidad de la República, Uruguai, e mestrado e doutorado em Política Científica e Tecnológica pela Unicamp. Professora Associada da Universidade Federal do Paraná (UFPR). E-mail: noela@ufpr.br
} 


\section{INTRODUÇÃO}

Os diferentes meios de comunicação, dentre eles a televisão, o jornal e os programas de TV pela internet, vêm estruturando espaços destinados à divulgação sistemática da ciência e da tecnologia. A análise das visões de tecnologia que circulam nesses espaços é relevante dado que estão entre os principais meios pelos quais o público leigo se informa sobre ciência e tecnologia (MCT, 2011). Ademais, a popularização da ciência e da tecnologia vem se tornando uma tarefa estimulada pelas agências de fomento de pesquisas (ASCOM do CNPq, 2012), como parte das atribuições dos pesquisadores, tarefa destacada anteriormente por Bourdieu (1997).

Nesse sentido, o propósito deste artigo ${ }^{3}$ é discutir o conceito de tecnologia a partir do caso da divulgação da nanotecnologia em três diferentes mídias, tomando como referência os Estudos Sociais da Ciência e da Tecnologia, em especial os Latinoamericanos.

A nanotecnologia é o desenvolvimento de aplicações e dispositivos que operam em escala molecular, a escala de um bilionésimo de metro ou $10^{-9}$. Além da escala, a novidade da nanotecnologia reside em pelo menos três características: 1) quanto mais reduzido o material, maior a sua superfície externa em relação à sua massa e a sua reatividade química; 2) as propriedades físicas, químicas e toxicológicas mudam quando os materiais são reduzidos à escala nanométrica; e 3) a possibilidade de aplicação de procedimentos bióticos em processos materiais ou de elementos materiais em organismos vivos. (FOLADORI; INVERNIZZI, 2006; GALLO, 2007).

A opção pela análise da abordagem de nanotecnologia justifica-se pela importância que as políticas públicas de ciência e tecnologia de diversos países, em todos os continentes, vêm atribuindo à mesma (HUNT, 2008; INVERNIZZI; FOLADORI, 2012), bem como ao destaque que vem recebendo em pesquisas de comunicação da ciência (MACNAGHTEN; GUIVANT, 2011; MARTINS; FERNANDES, 2011; INVERNIZZI; CAVICHIOLO, 2009; KURATH; GISLER, 2009; INVERNIZZI, 2008; AMORIM, 2008; PRIEST, 2008; FABER, 2006; STEPHENS, 2005). Além disso, a nanotecnologia é um tema emergente, em aberto, com tensões

\footnotetext{
${ }^{3}$ Uma versão inicial desse artigo foi apresentada no V Seminário Nacional Tecnologia e Sociedade, em Curitiba/PR, de 16 a 18 de outubro de 2013, com o título "O conceito de (nano)tecnologia em mídias".
} 
entre seu desenvolvimento e as preocupações do público (HUNT, 2008), o que permite captar posicionamentos de distintos grupos sociais no processo de sua configuração social.

A presente análise é parte das reflexões de tese de doutorado (KÖRBES, 2013) ${ }^{4}$, em que se analisa o conteúdo sobre nanotecnologia divulgado em três diferentes mídias brasileiras. Analisam-se as reportagens das seções de ciência do programa de TV aberta Bom Dia Brasil, das Organizações Globo, com cinco reportagens no período de 2008 a 2010; as seções de ciência do jornal Folha de S. Paulo, do Grupo Folha, com um total de 19 artigos publicados no mesmo período; e uma amostra não aleatória de $20 \%$ dos programas Nanotecnologia do Avesso, programa de entrevistas em webTV, promovido pela Rede de Pesquisa em Nanotecnologia, Sociedade e Meio Ambiente, totalizando 17 dos 86 programas exibidos no período de 2009 a 2010 (primeiros dois anos de realização do programa) e disponibilizados na internet ${ }^{5}$.

Para realizar a análise do material selecionado nos três meios de comunicação citados, utiliza-se a metodologia de análise de conteúdo, de cunho quantitativo e qualitativo (BARDIN, 1977), com fundamentação teórica explicitada a priori ou abordagem direcionada (MORAES, 1999). A análise de conteúdo é realizada por meio de análise categorial, em que se classificam as significações dos textos em categorias temáticas relacionadas ao objeto de pesquisa. Essa técnica segue as etapas de pré-análise, exploração do material ou codificação e tratamento dos resultados e interpretação (BARDIN, 1977).

A seguir, apresenta-se em uma única seção breves referenciais teóricos sobre tecnologia, seguidas de análise de algumas definições de nanotecnologia e reflexões finais sobre o conceito de (nano)tecnologia nas mídias analisadas.

\section{CONCEITOS E DEFINIÇÕES DE (NANO)TECNOLOGIA EM MÍDIAS}

Nesse trabalho, parte-se da compreensão de que a abordagem das três mídias sobre o conceito e as definições de nanotecnologia, stricto sensu, se vincula

\footnotetext{
${ }^{4}$ Agradecimento à Coordenação de Aperfeiçoamento de Pessoal de Nível Superior (CAPES) pela bolsa de estudos de doutorado.

${ }^{5}$ Neste artigo são listados apenas os materiais de divulgação científica citados diretamente. Para conferir a lista completa consulte Autora 1 (2013).
} 
à conceituação da tecnologia em sentido lato sensu, razão pela qual se utiliza o prefixo "nano" entre parênteses. Para a análise do conceito de (nano)tecnologia nas mídias selecionadas toma-se como pressuposto a perspectiva sociotécnica, que é "aquela que tentando superar as limitações dos determinismos lineares considera que as sociedades são tecnologicamente construídas ao mesmo tempo em que as tecnologias são socialmente configuradas" (THOMAS, 2011, p. 1, tradução nossa).

As ideias-chave da construção social da tecnologia enunciadas por Pinch (2008) partem dessa perspectiva de estreita relação entre a tecnologia e sociedade e por isso são essenciais para compreender o movimento de formação de visões de que participam as mídias e os atores nela representados. Sinteticamente, duas ideias são centrais nesse enfoque. A primeira é a flexibilidade interpretativa da tecnologia, ou seja, as tecnologias, especialmente nos seus primeiros estágios, estão abertas a mais de uma interpretação ou trajetória de desenvolvimento. A segunda é a importância atribuída aos marcos tecnológicos, noção semelhante à de paradigmas científicos, e à relação com os usuários.

Desse modo, um dos aspectos fundamentais para se compreender a construção do conceito de (nano)tecnologia nas mídias é o mapeamento dos atores relevantes que tomam parte desse processo social. Entende-se que os meios de comunicação social veiculam as visões de determinados atores, as quais são formadas em suas interações sociais, políticas e econômicas. Nesse processo, ao mesmo tempo em que as mídias difundem perspectivas de tecnologia de grupos sociais situados, pois como ressalta Bazzo (2011), a tecnologia não é um empreendimento neutro ou autônomo, atuam também como agentes formadores de visões sobre tecnologia.

Nesse sentido, questionamos: quais são os grupos sociais representados nos programas analisados? No Bom Dia Brasil e na Folha de S. Paulo predominam as vozes de pesquisadores das áreas de Ciências Exatas e da Saúde e das Engenharias. Em relação inversa, no Nanotecnologia do Avesso predominam os entrevistados (pesquisadores e não pesquisadores) das Ciências Humanas sobre essas áreas, as Exatas aparecem em segundo lugar, seguidas pela área de Ciências Sociais Aplicadas e Multidisciplinar, em terceiro lugar. Reconhece-se, assim, que cada mídia empodera alguns grupos (ou nós) da rede sociotécnica que constitui a (nano)tecnologia. 
Para entender as definições de nanotecnologia é necessário, portanto, examinar os interessados, as dinâmicas e as circunstâncias que levam à sua promulgação e estabilização. Conforme esclarecem Lacour e Vinck (2011), a escolha dos termos usados para designar um objeto é cuidadosamente ponderada pelos interessados, especialmente quando a regulamentação está em questão. Assim, as definições são uma construção social e podem se orientar a diferentes objetivos, como incentivar a inovação e o desenvolvimento tecnológico ou avaliar e gerenciar riscos potenciais.

Nos materiais analisados observaram-se algumas abordagens que favorecem os primeiros objetivos citados, ainda que não seja possível afirmar que houve um direcionamento deliberado para essa perspectiva. Um exemplo é a definição dimensional limitada ao escopo de 1 a 100nm, que segundo Lacour e Vinck (2011) interessa aos empresários e a muitos pesquisadores, pois encoraja a inovação e fortalece a adesão a projetos de pesquisa. Quando essa definição da nanotecnologia não é associada com outros aspectos, como a área de superfície e a mudança de comportamento dos materiais na nanoescala, a nanotecnologia pode ser entendida como algo bem delimitado, sem controvérsias. Isso acontece em grande parte das reportagens do Bom Dia Brasil e dos artigos da Folha de S. Paulo e raramente no Nanotecnologia do Avesso.

No entanto, é possível que nem sempre as definições assim apresentadas tenham a intencionalidade de favorecer os empresários ou grupos de pesquisadores. Como lembram Crone e Koch (2006) e Sánchez-Mora e Parga (2011) a escala invisível é o primeiro obstáculo epistemológico para compreender a nanotecnologia. Certamente essa é uma das razões pelas quais a definição de escala nanométrica está presente em quase todos os materiais analisados, o problema é que poucas vezes vem acompanhada de uma contextualização.

As definições da nanotecnologia também podem se orientar aos objetivos de avaliação e monitoramento de riscos potenciais. Por exemplo, Leite (2009), ao enfatizar o termo nanopartículas e associá-las com nanoparticularidades, evidencia que as propriedades mudam radicalmente na escala nanométrica e vincula essa definição à necessidade de regulamentação da nanotecnologia. Este artigo, junto com outros dois do mesmo autor (LEITE, 2008a; LEITE, 2008b), são os únicos da Folha de S. Paulo que tiveram como assunto central os riscos e as implicações 
legais, sociais e éticas da nanotecnologia. Dois possíveis fatores podem explicar essa condição, que são a proximidade do jornalista com os Estudos Sociais da Ciência e da Tecnologia e a possibilidade de que os artigos se situam em seção destinada ao público mais informado. Essa seção parece se afastar do jornalismo commodity (indiferenciado e de massa), expressão usada pelo diretor de redação do jornal José Frias Filho para se referir às tendências da reformulação editorial que suprimiu a seção Mais Ciência em meados de 2010 (ANDRADE, 2010).

A nanotecnologia também foi definida em relação à sua novidade ou continuidade de processos já existentes na natureza. Dentre todos os meios analisados, essa tensão ficou mais evidente no Bom Dia Brasil. Por um lado, o telejornal destaca as novidades da nanotecnologia associando-as com a criação de novos objetos materiais, novos produtos com novas funcionalidades e novos serviços com perspectivas de efeitos positivos sobre a competitividade das empresas, a qualidade de vida, a saúde e a preservação do meio ambiente. Essa visão também foi observada na Folha de S. Paulo e manifesta a recorrência (talvez pela aderência a necessidades similares de mercado, pautadas pela concorrência capitalista) à atmosfera de prosperidade e utopias e a um discurso muito semelhante feito mais de 100 anos antes nas Exposições Universais ${ }^{6}$ e Passagens ${ }^{7}$ na Europa: o discurso do progresso tecnológico e do determinismo tecnológico que transparece na associação linear entre nova tecnologia e benefícios econômicos e sociais. Isso se repete apesar dos avanços, tanto no campo acadêmico como no social, no sentido de questionar essas concepções.

Por outro lado, são desenvolvidos no telejornal os argumentos de que nem a medida, nem a matéria em escala nanométrica são novas, que elas já estão presentes há muito tempo nos medicamentos (LOSEKANN; GILZ, 2009) e nos vitrais das igrejas antigas, e que a nanotecnologia é natural (DUARTE; ARAÚJO, 2009a), pois sua dimensão está presente nas plantas, na poeira, nas proteínas, nos vírus e na molécula de DNA. A aparente contradição - pois de fato houve uso empírico da nanotecnologia no passado e há inúmeros processos em escala nanométrica na

\footnotetext{
${ }^{6}$ As Exposições Universais foram fenômenos típicos do século XIX que visavam o contato entre produtores e consumidores para divulgar ou anunciar a existência de novos produtos e ampliar as vendas (PESAVENTO, 1997). Para uma análise crítica dessas exposições e das Passagens, sugerese Benjamin (2006) e Pesavento (1997).

7 As Passagens são espécies de galerias da primeira metade do século XIX em Paris, na França, também conhecidas como "avós dos shopping-centers".
} 
natureza - poderia resultar da intenção de reforçar que a nanotecnologia é inofensiva, fortalecer a adesão a projetos de pesquisa e o respectivo financiamento público, evitando a ansiedade diante do novo e o debate sobre riscos gerado sobre outras tecnologias emergentes.

No programa Nanotecnologia do Avesso, sob diferentes perspectivas, vários entrevistados reconhecem que a nanotecnologia cria nova fronteira para a indústria em diversos ramos e, aliado a isso, novas soluções ou novos problemas para a sociedade (GAMA, 2009; JARDIM, 2009; JENSEN; VIEIRA, 2010; RATTNER, 2009; SALOMÃO, 2009; MINOZZI, 2010, NORDMANN, 2010; BENSAUDE-VINCENT, 2010), tais como: os riscos para a saúde de trabalhadores e de consumidores; os riscos para o meio ambiente e as diversas formas de vida; e os riscos econômicos de investimento em uma tecnologia emergente e seus efeitos sobre o emprego. Esse cenário motiva a demanda pela regulação obrigatória da nanotecnologia e a adoção do princípio de precaução (RATTNER, 2009; NORDMANN, 2010; BLAU, 2009). Desse modo, o programa discute com maior amplitude a configuração social da (nano)tecnologia, os conflitos de interesse e a sua não neutralidade, ressaltando visões que podem ser qualificadas como distópicas, pois discutem condições de vida adversas, como a opressão, a exclusão social e poluição ambiental.

\section{CONSIDERAÇÕES FINAIS}

A análise da abordagem de (nano)tecnologia em três diferentes mídias evidencia que as definições dependem de quem as produz e das circunstâncias que levam à sua promulgação e estabilização. No Bom Dia Brasil e na Folha de S. Paulo o conceito de nanotecnologia enfatiza o artefato material e o potencial das novas propriedades e funções da matéria em nanoescala para a inovação. Assim, explicita minimamente sua configuração social por uma rede sociotécnica e reproduz discursos baseados em modelos lineares, como a suposta neutralidade, inexorabilidade e progresso contínuo da ciência e da tecnologia. No jornal Folha de S. Paulo a abordagem mais alinhada à perspectiva sociotécnica está situada em seção que parece estar destinada aos leitores mais interessados em assuntos científicos. Já no Nanotecnologia do Avesso, por outro lado, é proeminente a discussão de potenciais riscos e implicações sociais, legais e éticas da 
nanotecnologia, e a demanda pela aplicação do princípio de precaução e regulação obrigatória.

Esse comparativo permite concluir que grupos relevantes com perspectivas distintas de (nano)tecnologia incidem desigualmente na formação da opinião pública e no direcionamento das políticas públicas de (nano)tecnologia. Os grupos de atores favorecidos pelo Bom Dia Brasil e pela Folha de S. Paulo sustentam a estabilização da nanotecnologia para atender interesses de parte da comunidade científica e do mercado, e os atores privilegiados pelo Nanotecnologia do Avesso assumem a flexibilidade e instabilidade dessa tecnologia, buscando modificá-la mediante suas demandas de adoção do princípio de precaução e de regulação obrigatória.

Concluindo, coexistem nas mídias analisadas perspectivas que pendem para a utopia ou a distopia ao tratar da tecnologia e, em particular, da nanotecnologia. Todas as mídias descrevem as novas propriedades e funções da matéria em nanoescala, mas diferem na forma de fazê-lo, de acordo com os interesses dos atores relevantes representados: ora salientam o potencial de inovação que tais propriedades geram, associando-as com uma atmosfera de prosperidade e utopia; ora novos riscos associados a elas, ressaltando a distopia e a discussão de formas de exclusão, opressão e poluição potencializadas pela nanotecnologia.

\section{REFERÊNCIAS}

AMORIM, Tade-Ane de. Nanotecnologia na imprensa: análise de conteúdo do jornal Folha de São Paulo. Em Tese. Revista eletrônica dos pós-graduandos em Sociologia Política da UFSC, v. 4, n. 2, p. 20-36, jan./jul. 2008.

ANDRADE, Fernando G. 0 jornal do futuro. Documentário. São Paulo: Spray Filmes, 2010.

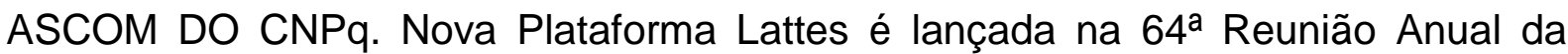
SBPC. Jornal da Ciência e-mail, Rio de Janeiro, ed. 4548, 26 de jul. 2012.

BARDIN, Laurence. Análise de conteúdo. Lisboa, Portugal: Edições 70, 1977.

BAZZO, Walter A. Ciência, tecnologia e sociedade e o contexto da educação tecnológica. 3. ed. rev. Florianópolis: Ed. da UFSC, 2011.

BENJAMIN, Walter. Passagens. Tradução: Irene Aron. Belo Horizonte: UFMG, 2006. 
BENSAUDE-VINCENT, Bernadette. Entrevista. Nanotecnologia do Avesso, São Paulo, 14 set. 2010. Programa 082. Disponível em: <http://vimeo.com/14781320>. Acesso em: 08 jul. 2012.

BLAU, Judith. Entrevista. Nanotecnologia do Avesso, São Paulo, 18 maio 2009. Programa 018. Disponível em: <http://vimeo.com/4847358>. Acesso em: 08 jul. 2012.

BOURDIEU, Pierre. Sobre a televisão seguido de A influência do jornalismo e os Jogos Olímpicos. Tradução: Maria Lúcia M. Rio de Janeiro: Jorge Zahar Ed., 1997.

CRONE, Wendy C.; $\mathrm{KOCH}$, Susan E. Bringing nano to the public: a collaboration opportunity for researchers and museums. NISE Network. Science Museum of Minnesota, St.Paul, Minnesota, 2006.

DUARTE, Neide; ARAÚJO, Wilson. Nanotecnologia transforma vidas sem que se perceba. Rede Globo: Bom Dia Brasil, Série Universo Nano, 2009a. Disponível em: <http://g1.globo.com/bomdiabrasi//0,16020-p-01092009,00.html>. Acesso em: 01 set. 2009.

FABER, Brenton. Popularizing Nanoscience: The Public Rhetoric of Nanotechnology, 1986-1999, Technical Communication Quarterly, [S. I], v.15, n. 2, p. 141-169, 2006.

FOLADORI, Guillermo; INVERNIZZI, Noela. La nanotecnología: una solución en busca de problemas. Comércio Exterior, México, DF, v. 56, n. 4, p. 326-334, abr. 2006.

GALLO, Jairo G. Historia lejana y reciente de la mayor revolución em la historia. In: GALLO, Jairo G.; GONZALEZ, Edgar; GÓRNEZ-BAQUERO, Fernando. (Eds.). Nanotecnociencia: nociones preliminares sobre el universo nanoscópico. Colombia: Buinaima, 2007, p. 25-68.

GAMA, Cátia F. Entrevista. Nanotecnologia do Avesso, São Paulo, 13 abr. 2009. Programa 013. Disponível em: <http://vimeo.com/21106807>. Acesso em: 08 jul. 2012.

HUNT, Geoffrey. Nanotechnologies and society in Europe. In: HUNT, Geoffrey; MEHTA, Michael D. (Eds.). Nanotechnology risk, ethics and law. 2. ed. London; Sterling, VA: Earthscan, p. 92-104, 2008.

INVERNIZZI, Noela; FOLADORI, Guillermo. ¿Hacia donde van las nanotecnologías en América Latina? In: FOLADORI, Guillermo; INVERNIZZI, Noela; ZÁYAGO, Edgar; (Coords.) Perspectivas sobre el desarrollo de las nanotecnologías en América Latina. Mexico, DF: M.A. Porrúa, 2012, p. 229-233.

INVERNIZZI, Noela. Visions of Brazilian Scientists on Nanosciences and Nanotechnologies. NanoEthics, [New York?], v. 2, n. 2, p. 133-148, 2008. 
INVERNIZZI, Noela; CAVICHIOLO, Cibele. ¿Nanotecnología en los medios: qué información llega al público? Redes, Buenos Aires, v. 15, n. 29, p. 139-175, maio 2009.

JARDIM, Fernando R. Entrevista. Nanotecnologia do Avesso, São Paulo, 16 nov. 2009. Programa 043. Disponível em: <http://vimeo.com/7795675>. Acesso em: 08 jul. 2012.

JENSEN, Thomaz F.; VIEIRA FILHO, Antonio G. Entrevista. Nanotecnologia do Avesso, São Paulo, 29 jun. 2010. Programa 071. Disponível em: <http://vimeo.com/12929387>. Acesso em: 08 jul. 2012.

KÖRBES, Clecí. Educação não-formal em mídias: divulgação científica sobre nanotecnologia. 319 f. Tese (Doutorado em Tecnologia) - Programa de PósGraduação em Tecnologia, Universidade Tecnológica Federal do Paraná, Curitiba, 2013. Disponível em: <http://repositorio.utfpr.edu.br/jspui/handle/1/491>. Acesso em: 26 jun. 2013.

Educação não-formal e informal em ciência e tecnologia: divulgação científica e formação de opinião sobre reprodução assistida e seus desdobramentos na Folha de S. Paulo. 2008. 176 f. Dissertação (Mestrado em Educação) - Programa de Pós-Graduação em Educação, Universidade Federal do Paraná, Curitiba, 2008.

KURATH, Monika; GISLER, Priska. Informing, involving or engaging? Science communication, in the ages of atom-, bio- and nanotechnology. Public Understanding of Science, [London?], v. 18, n. 5, p. 559-573, 2009.

LACOUR, Stéphanie; VINCK, Dominique. Nanoparticles, nanomaterials, what are we talking about? Socio-legal views on constructing the object of regulation in the field of "nano" risks. Paris: INRS, 2011.

LEITE, Marcelo. Nanotubos, amianto e câncer. Folha de S. Paulo, São Paulo, 25 maio 2008a. Mais Ciência, p. 9.

Nanodemocracia. Folha de S. Paulo, São Paulo, 17 ago. 2008b. Mais Ciência, p. 3.

Nanoparticularidades. Folha de S. Paulo, São Paulo, 27 dez. 2009. Mais Ciência, p. 3.

LOSEKANN, Marcos; GILZ, Sérgio. Nanotecnologia é esperança na cura de doenças como aids e câncer. Rede Globo: Bom Dia Brasil, Série Universo Nano, 2009. Disponível em: <http://g1.globo.com/bomdiabrasil/0,,16020-p03092009,00.html>. Acesso em: 03 set. 2009.

MACNAGHTEN, Phil; GUIVANT, Julia S. Converging citizens? Nanotechnology and the political imaginary of public engagement in Brazil and the United Kingdom, Public Understanding of Science, [London?], v. 20, n. 2, p. 207-220, 2011. 
MARTINS, Paulo R.; FERNANDES, Maria F. M. Nanotecnologia do Avesso: uma experiência de engajamento público em ciência e tecnologia. Revista Brasileira de Ciência, Tecnologia e Sociedade, São Carlos, v. 2, n. 1, p. 109-119, jan./jun. 2011.

MCT - Ministério da Ciência e da Tecnologia. Percepção Pública da Ciência e Tecnologia no Brasil. Resultados da enquete de 2010. Brasília/DF, 2011.

MINOZZI, Daniel T. Entrevista. Nanotecnologia do Avesso, São Paulo, 27 jul. 2010. Programa 075. Disponível em: <http://vimeo.com/14048055>. Acesso em: 08 jul. 2012.

MORAES, Roque. Análise de conteúdo. Revista Educação, Porto Alegre, v. 22, n. 37, p. 7-32, 1999.

PESAVENTO, Sandra J. Exposições universais. São Paulo: Cultrix, 1997.

PINCH, Trevor. La tecnología como institución: ¿Qué nos pueden enseñar los estudios sociales de la tecnología? Redes, Buenos Aires, v. 14, n. 27, p. 77-96, 2008.

PRIEST, Susanna. Biotechnology, nanotechnology, media, and public opinion. In: DAVID, Kenneth H.; THOMPSON, Paul B. (eds.). What Can Nanotechnology Learn From Biotechnology?: Social and Ethical Lessons for Nanoscience From the Debate Over Agrifood Biotechnology and GMOs. Amsterdan; Boston: Elsevier/Academic Press, 2008.

RATTNER, Henrique. Entrevista. Nanotecnologia do Avesso, São Paulo, 02 mar. 2009. Programa 007. Disponível em: <http://vimeo.com/21670345>. Acesso em: 08 jul. 2012.

SALOMÃO, Rafael. Entrevista. Nanotecnologia do Avesso, São Paulo, 29 jun. 2009. Programa 024. Disponível em: <http://vimeo.com/5510078>. Acesso em: 08 jul. 2012.

SÁNCHEZ-MORA, M. Carmen; PARGA, Julia T. El manejo de las escalas como obstáculo epistemológico en la divulgación de la nanociencia. Mundo Nano Revista Interdisciplinaria en nanociencia y nanotecnología, Ciudad de Mexico, v. 4, n. 2, p. 83-102, jul-dic./2011.

STEPHENS, Lowndes F. News Narratives about Nano S\&T in Major U.S. and NonU.S. Newspapers. Science Communication, [New York?], v. 27, n. 2, p. 175-199, dec./2005.

THOMAS, Hernán. Tecnologías sociales y ciudadanía sócio-tecnica. Notas para la construcción de la matriz material de um futuro viable. Ciência \& Tecnologia Social, Brasília/DF, v. 1, n. 1, jul. 2011. 